\title{
Local residues of holomorphic 1-forms on an isolated surface singularity
}

\author{
Oliver Klehn \\ Institut für Mathematik, Universität Hannover, \\ Postfach 6009, D-30060 Hannover, Germany \\ E-mail: klehn@math.uni-hannover.de
}

\begin{abstract}
We define local residues of holomorphic 1-forms on an isolated surface singularity that have isolated zeros and prove that a certain residue equals the index of the 1-forms defined by Ebeling and Gusein-Zade.
\end{abstract}

\section{Introduction}

Let $f_{1}, \ldots, f_{n} \in \mathcal{O}_{\mathbb{C}^{n}, 0}$ define a regular sequence, let $J_{f}$ be the determinant of the Jacobian matrix of $f:=\left(f_{1}, \ldots, f_{n}\right)$ and set

$$
Q_{f}:=\frac{\mathcal{O}_{\mathbb{C}^{n}, 0}}{\left(f_{1}, \ldots, f_{n}\right)} .
$$

Then a classical result states

$$
\operatorname{ind}_{\mathbb{C}^{n}, 0} f=\operatorname{dim}_{\mathbb{C}} Q_{f}=\operatorname{res}_{\mathbb{C}^{n}, 0}\left[\begin{array}{c}
J_{f} \\
f_{1} \ldots f_{n}
\end{array}\right],
$$

where ind is the Poincaré-Hopf index and res the local residue symbol. Recall that the residue is defined for any $h \in \mathcal{O}_{\mathbb{C}^{n}, 0}$ by

$$
\operatorname{res}_{\mathbb{C}^{n}, 0}\left[\begin{array}{c}
h \\
f_{1} \ldots f_{n}
\end{array}\right]:=\frac{1}{(2 \pi i)^{n}} \int_{\Gamma} \frac{h d z_{1} \wedge \cdots \wedge d z_{n}}{f_{1} \ldots f_{n}}
$$

where $\Gamma$ is the real $n$-cycle $\left\{\left|f_{i}\right|=\epsilon_{i}, i=1, \ldots, n\right\}$ for $\epsilon_{i} \in \mathbb{R}_{>0}$ chosen small enough oriented so that $d\left(\arg f_{1}\right) \wedge \cdots \wedge d\left(\arg f_{n}\right) \geq 0$, and the Poincaré-Hopf index is the degree of the map $f /\|f\|$ restricted to a small sphere at the origin, see also [1, [1, 11] for the definitions.

In the framework of singularity theory it arises a natural question: How to generalize these definitions when one considers holomorphic function germs on an isolated singularity $(V, 0) \subset\left(\mathbb{C}^{n}, 0\right)$ ? There are two directions one can go. The first is the definition of an index of holomorphic vector fields tangent to $(V, 0)$, called the GSV index. The results can be found in 2 , $4,0,8,9,10$, 12. The other direction is to consider holomorphic 1-forms on $(V, 0)$ which has been done by Ebeling and Gusein-Zade in [3]. This is the situation we give a 
generalization of the residue symbol and therefore we briefly recall the main results of [3].

Let $(V, 0)=\left(\left\{f_{1}=\cdots=f_{q}=0\right\}, 0\right) \subset\left(\mathbb{C}^{n}, 0\right)$ be an isolated complete intersection singularity and $\omega=\sum_{i=1}^{n} \omega_{i} d z_{i}$ the germ of a holomorphic 1-form on $\left(\mathbb{C}^{n}, 0\right)$ which has an isolated zero on $(V, 0)$. Choose a sufficiently small sphere $S_{\delta}$ around the origin in $\mathbb{C}^{n}$ which intersects $V$ transversally and consider the link $K=V \cap S_{\delta}$ of $V$. The 1-forms $\omega, d f_{1}, \ldots, d f_{q}$ are linearly independent for all points of $K$ and we have a well defined map

$$
\left(\omega, d f_{1}, \ldots, d f_{q}\right): K \rightarrow W_{q+1}\left(\mathbb{C}^{n}\right)
$$

where $W_{q+1}\left(\mathbb{C}^{n}\right)$ denotes the manifold of $(q+1)$-frames in the dual $\mathbb{C}^{n}$. We have

$$
H_{2 n-2 q-1}(K) \cong \mathbb{Z}, \quad H_{2 n-2 q-1}\left(W_{q+1}\left(\mathbb{C}^{n}\right)\right) \cong \mathbb{Z}
$$

and therefore the map has a degree. We let $K$ to be oriented as boundary of the complex manifold $V \backslash\{0\}$ here. The index ind ${ }_{V, 0} \omega$ of $\omega$ is defined to be the degree of this map. (If $V$ is a curve $K$ can have more components, we will sum over the degrees of the components then.)

Let $J$ be the ideal in $\mathcal{O}_{\mathbb{C}^{n}, 0}$ generated by $f_{1}, \ldots, f_{q}$ and the $(q+1)$-minors of the matrix

$$
\left(\begin{array}{ccc}
\frac{\partial f_{1}}{\partial z_{1}} & \cdots & \frac{\partial f_{1}}{\partial z_{n}} \\
\vdots & \ddots & \vdots \\
\frac{\partial f_{q}}{\partial z_{1}} & \cdots & \frac{\partial f_{q}}{\partial z_{n}} \\
\omega_{1} & \cdots & \omega_{n}
\end{array}\right)
$$

For a regular value $0 \neq \epsilon=\left(\epsilon_{1}, \ldots, \epsilon_{q}\right) \in \mathbb{C}^{q}$ of $f$ chosen small enough and a small ball $B_{\delta}$ around the origin in $\mathbb{C}^{n}$ define $V_{\epsilon}:=f^{-1}(\epsilon) \cap B_{\delta} . V_{\epsilon}$ is transversally to $S_{\delta}$ then. We set $\mathcal{A}:=\mathcal{O}_{\mathbb{C}^{n}, 0} / J$ and have the following theorem of Ebeling and Gusein-Zade:

Theorem 1. (i) If $(V, 0)$ is smooth, ind $_{V, 0} \omega$ is the usual Poincaré-Hopf index. (ii) ind $_{V, 0} \omega$ equals the number of zeros of $\omega$ on $V_{\epsilon}$ counted with multiplicities.

(iii) $\operatorname{ind}_{V, 0} \omega=\operatorname{dim}_{\mathbb{C}} \mathcal{A}$.

In this paper we define if $V$ is a surface a linear form

$$
\operatorname{res}_{V, 0}: \mathcal{A} \rightarrow \mathbb{C},
$$

which we call the relative residue form of $\omega$ and prove that for a certain class $\sigma \in \mathcal{A}$ one has a formula

$$
\operatorname{ind}_{V, 0} \omega=\operatorname{res}_{V, 0}(\sigma) .
$$

We use the linear form to prove some algebraic properties of $\mathcal{A}$. There is used classical Grothendieck residue theory and we will therefore recall the main facts as them can be found in $[7$. With the notations introduced at the beginning of this section we summarize some results of [7] in the following theorem: 
Theorem 2. (i) For $i=1, \ldots, n$ let $g_{i}:=\sum_{j=1}^{n} a_{i j} f_{j}$ so that $g_{1}, \ldots, g_{n}$ is a regular sequence and let $A$ be the matrix $\left(a_{i j}\right)_{i=1, \ldots, n}^{j=1, \ldots, n}$. Then one has for any $h \in \mathcal{O}_{\mathbb{C}^{n}, 0}$

$$
\operatorname{res}_{\mathbb{C}^{n}, 0}\left[\begin{array}{c}
h \\
f_{1} \ldots f_{n}
\end{array}\right]=\operatorname{res}_{\mathbb{C}^{n}, 0}\left[\begin{array}{c}
h \operatorname{det} A \\
g_{1} \ldots g_{n}
\end{array}\right] .
$$

(ii) The residue defines a linear form

$$
\operatorname{res}_{\mathbb{C}^{n}, 0}\left[\begin{array}{c}
\cdot \\
f_{1} \ldots f_{n}
\end{array}\right]: Q_{f} \rightarrow \mathbb{C} .
$$

(iii) The induced pairing

$$
B: Q_{f} \times Q_{f} \rightarrow \mathbb{C}
$$

defined by

$$
B(h, g):=\operatorname{res}_{\mathbb{C}^{n}, 0}\left[\begin{array}{c}
h \cdot g \\
f_{1} \ldots f_{n}
\end{array}\right]
$$

is non-degenerate.

(iv) For perturbations $f_{\epsilon}$ and $h_{\epsilon}$ of $f$ and $h$ one has

$$
\operatorname{res}_{\mathbb{C}^{n}, 0}\left[\begin{array}{c}
h \\
f_{1} \ldots f_{n}
\end{array}\right]=\lim _{\epsilon \rightarrow 0} \sum_{i} \operatorname{res}_{\mathbb{C}^{n}, p_{i}}\left[\begin{array}{c}
h_{\epsilon} \\
f_{\epsilon, 1} \ldots f_{\epsilon, n}
\end{array}\right]
$$

where one sums over the zeros of $f_{\epsilon}$ in a neighbourhood of the origin.

We want to formulate our main result. Let $q=n-2$ and $M_{i}$ the matrix obtained from

$$
\left(\begin{array}{ccc}
\frac{\partial f_{1}}{\partial z_{1}} & \cdots & \frac{\partial f_{1}}{\partial z_{n}} \\
\vdots & \ddots & \vdots \\
\frac{\partial f_{n-2}}{\partial z_{1}} & \cdots & \frac{\partial f_{n-2}}{\partial z_{n}} \\
\omega_{1} & \cdots & \omega_{n}
\end{array}\right)
$$

by cancelling the $i$-th column, $m_{i}:=\operatorname{det}\left(M_{i}\right)$ and $M:=\left((-1)^{i} \frac{\partial m_{i}}{\partial z_{j}}\right)$. Let $\sigma$ be the coefficient of $t^{n-2}$ in the characteristical polynomial of $M$. We will prove that there exits a linear change of coordinates (in fact a generic one) so that $\left(m_{1}, m_{2}\right)$ is a regular $\mathcal{O}_{V, 0}$-sequence and call these coordinates good coordinates. Moreover we define for $h \in \mathcal{O}_{\mathbb{C}^{n}, 0}$

$$
\operatorname{res}_{V, 0}\left[\begin{array}{c}
h \\
m_{1} m_{2}
\end{array}\right]:=\frac{1}{(2 \pi i)^{2}} \int_{\Sigma} \frac{h d z_{1} \wedge d z_{2}}{m_{1} m_{2}}
$$

with $\Sigma:=\left\{f_{1}=\cdots=f_{n-2}=0,\left|m_{1}\right|=\delta_{1},\left|m_{2}\right|=\delta_{2}\right\}$ oriented so that $d\left(\arg m_{1}\right) \wedge d\left(\arg m_{2}\right) \geq 0$ and where $\delta_{1}, \delta_{2}$ are small positive real numbers. The following theorem is our main result: 
Theorem 3. In each system of good coordinates we have a linear form

$$
\operatorname{res}_{V, 0}\left[\begin{array}{c}
\cdot \\
m_{1} m_{2}
\end{array}\right]: \mathcal{A} \rightarrow \mathbb{C}
$$

with

$$
\operatorname{dim}_{\mathbb{C}} \mathcal{A}=\operatorname{res}_{V, 0}\left[\begin{array}{c}
\sigma \\
m_{1} m_{2}
\end{array}\right] .
$$

I wish to thank W. Ebeling and S.M. Gusein-Zade for useful discussions and the referee for useful comments concerning the presentation. The idea that one should try to find a residue formula for the index of a holomorphic 1-form is due to W. Ebeling.

\section{Absolute and relative residues}

Let $(V, 0) \subset\left(\mathbb{C}^{n}, 0\right)$ be an icis as before and $g_{1}, \ldots, g_{n-q} \in \mathcal{O}_{\mathbb{C}^{n}, 0}$ define an isolated zero on $(V, 0)$. If the real hypersurfaces $\left\{\left|g_{i}\right|=\delta_{i}\right\}$ for small $\delta_{i}$ are in general position let

$$
\Sigma:=\left\{f_{1}=\cdots=f_{q}=0,\left|g_{i}\right|:=\delta_{i}, i=1, \ldots, n-q\right\}
$$

to be the real $(n-q)$-cycle oriented so that $d\left(\arg g_{1}\right) \wedge \cdots \wedge d\left(\arg g_{n-q}\right) \geq 0$. Then we have for each

$$
\eta \in \Omega_{V, 0}^{n-q}:=\Omega_{\mathbb{C}^{n}, 0}^{n-q} /\left(\Sigma f_{i} \Omega_{\mathbb{C}^{n}, 0}^{n-q}+\Sigma d f_{i} \wedge \Omega_{\mathbb{C}^{n}, 0}^{n-q-1}\right)
$$

a well defined integral

$$
\frac{1}{(2 \pi i)^{n-q}} \int_{\Sigma} \frac{\eta}{g_{1} \ldots g_{n-q}}
$$

which we denote by

$$
\operatorname{res}_{V, 0}\left[\begin{array}{c}
\eta \\
g_{1} \ldots g_{n-q}
\end{array}\right]
$$

We now want to prove a relation between these relative residues and the absolute residues. Define

$$
\lambda: \Omega_{V, 0}^{n-q} \rightarrow \mathcal{O}_{V, 0}
$$

by $\lambda(\eta):=h$, where $h$ is defined by

$$
h d z_{1} \wedge \cdots \wedge d z_{n}:=\eta \wedge d f_{1} \wedge \cdots \wedge d f_{q} .
$$

We want to prove the following theorem:

Theorem 4.

$$
\operatorname{res}_{V, 0}\left[\begin{array}{c}
\eta \\
g_{1} \ldots g_{n-q}
\end{array}\right]=\operatorname{res}_{\mathbb{C}^{n}, 0}\left[\begin{array}{c}
\lambda(\eta) \\
g_{1} \ldots g_{n-q} f_{1} \ldots f_{q}
\end{array}\right] .
$$


The intersection multiplicity $I$ of the hypersurfaces defined by $f_{1}, \ldots, f_{q}$, $g_{1}, \ldots, g_{n-q}$ is also given as a relative integral:

\section{Corollary 1.}

$$
I=\frac{1}{(2 \pi i)^{n-q}} \int_{\Sigma} \frac{d g_{1} \wedge \cdots \wedge d g_{n-q}}{g_{1} \ldots g_{n-q}} .
$$

The Theorem follows from the next Lemma if we set

$$
D F:=\operatorname{det}\left(\frac{\partial\left(f_{1}, \ldots, f_{q}\right)}{\partial\left(z_{n-q+1}, \ldots, z_{n}\right)}\right) .
$$

\section{Lemma 1.}

$$
\operatorname{res}_{V, 0}\left[\begin{array}{c}
h d z_{1} \wedge \cdots \wedge d z_{n-q} \\
g_{1} \ldots g_{n-q}
\end{array}\right]=\operatorname{res}_{\mathbb{C}^{n}, 0}\left[\begin{array}{c}
h \cdot D F \\
g_{1} \ldots g_{n-q} f_{1} \ldots f_{q}
\end{array}\right] .
$$

Proof of Theorem 国. Let $1 \leq i_{1}<\cdots<i_{n-q} \leq n$ and $1 \leq j_{1}<\cdots<j_{q} \leq n$ the complement, $\sigma:=\left(i_{1}, \ldots, i_{n-q}, j_{1}, \ldots, j_{q}\right) \in S_{n}$. Then we get

$$
\operatorname{res}_{V, 0}\left[\begin{array}{c}
h d z_{i_{1}} \wedge \ldots \wedge d z_{i_{n-q}} \\
g_{1} \ldots g_{n-q}
\end{array}\right]=\operatorname{sign} \sigma \cdot \operatorname{res}_{\mathbb{C}^{n}, 0}\left[\begin{array}{c}
h \cdot \frac{\partial\left(f_{1}, \ldots, f_{q}\right)}{\partial\left(z_{j_{1}}, \ldots, z_{j_{q}}\right)} \\
g_{1} \ldots g_{n-q} f_{1} \ldots f_{q}
\end{array}\right]
$$

by Lemma 1 using the permutation of coordinates $\sigma$ and the integral transformation formula. Now the theorem follows from equation 1 using the Laplace expansion formula.

First we prove a special case of Lemma 1. Define

$$
F:=\left(g_{1}, \ldots, g_{n-q}, f_{1}, \ldots, f_{q}\right) .
$$

Claim 1. Lemma 1 holds if 0 is a regular value of $F$.

To prove this we need some facts from linear algebra.

Lemma 2. Let $H:=\left(\begin{array}{cc}A & B \\ C & D\end{array}\right)$ be the decomposition of a $n \times n$-matrix in four blocks where $A$ and $D$ are squared and $A$ is invertible. Then

$$
\operatorname{det} H=\operatorname{det} A \cdot \operatorname{det}\left(D-C A^{-1} B\right) .
$$

Proof. It is just a simple exercise.

Lemma 3. Let $H:=\left(\begin{array}{cc}A & B \\ C & D\end{array}\right)$ be an invertible $n \times n$-matrix with $H^{-1}=$ $\left(\begin{array}{cc}E & F \\ G & I\end{array}\right)$ where $A$ und $E$ are $j \times j$-matrices and $D$ and $I$ are $(n-j) \times(n-j)$ matrices. Then

$$
\operatorname{det} D=\operatorname{det} E \cdot \operatorname{det} H \text {. }
$$


Proof. We have

$$
1=H H^{-1}=\left(\begin{array}{cc}
A E+B G & A F+B I \\
C E+D G & C F+D I
\end{array}\right)
$$

Let $E$ be invertible. Sice we have $C E+D G=0$ we get $-C F-D G E^{-1} F=0$ and therefore with $D I=1-C F$ it follows

$$
\operatorname{det}\left(1-C F-D G E^{-1} F\right)=\operatorname{det} D \cdot \operatorname{det}\left(I-G E^{-1} F\right)=1 .
$$

This means that $D$ is invertible. Now assume that $D$ is invertible. Since we have $C E+D G=0$ we get $B D^{-1} C E+B G=0$. From $1-B G=A E$ it follows that

$$
\operatorname{det}\left(1-B D^{-1} C E-B G\right)=\operatorname{det}\left(A-B D^{-1} C\right) \cdot \operatorname{det} E=1
$$

and so we find that $E$ must be invertible. We have shown that $E$ is invertible if and only if $D$ is invertible and so the Lemma follows for non invertible $E$. If $E$ is invertible we get by Lemma 2

$$
\operatorname{det} H^{-1}=\operatorname{det} E \cdot \operatorname{det}\left(I-G E^{-1} F\right) .
$$

By equation 2 we get

$$
\operatorname{det} H^{-1}=\operatorname{det} E \cdot \frac{1}{\operatorname{det} D}
$$

and $\operatorname{det} D=\operatorname{det} E \cdot \operatorname{det} H$ follows.

Proof of the Claim. We find that $V$ is smooth and 0 is also a regular value of $G:=\left.\left(g_{1}, \ldots, g_{n-q}\right)\right|_{V}$. We choose new coordinates $x:=F(z)$ on $\left(\mathbb{C}^{n}, 0\right)$ resp. $x^{\prime}:=G(z)$ on $(V, 0)$ and set $\tilde{F}:=F^{-1}$ resp. $\tilde{G}:=G^{-1}$. Using the integral transformation formula and the Cauchy integral formula we get

$$
\begin{aligned}
\operatorname{res}_{V, 0}\left[\begin{array}{c}
h d z_{1} \wedge \ldots \wedge d z_{n-q} \\
g_{1} \ldots g_{n-q}
\end{array}\right] & =\frac{1}{(2 \pi i)^{n-q}} \int_{\Sigma} \frac{h d z_{1} \wedge \cdots \wedge d z_{n-q}}{g_{1} \ldots g_{n-q}} \\
& =\frac{1}{(2 \pi i)^{n-q}} \int_{T} \frac{h(\tilde{G}) \frac{\partial\left(\tilde{G}_{1}, \ldots, \tilde{G}_{n-q}\right)}{\partial\left(x_{1}, \ldots, x_{n-q}\right)}}{x_{1} \ldots x_{n-q}} \wedge \cdots \wedge d x_{n-q} \\
& =h(\tilde{G}(0)) \frac{\partial\left(\tilde{G}_{1}, \ldots, \tilde{G}_{n-q}\right)}{\partial\left(x_{1}, \ldots, x_{n-q}\right)}(0) \\
& =h(0) \frac{\partial\left(\tilde{F}_{1}, \ldots, \tilde{F}_{n-q}\right)}{\partial\left(x_{1}, \ldots, x_{n-q}\right)}(0) .
\end{aligned}
$$

$T$ is the torus

$$
T:=\left\{\left|x_{i}\right|=\delta_{i}, i=1, \ldots, n-q\right\}
$$

oriented as usually here. Similarly we get

$$
\operatorname{res}_{\mathbb{C}^{n}, 0}\left[\begin{array}{c}
h \cdot D F \\
g_{1} \ldots g_{n-q} f_{1} \ldots f_{q}
\end{array}\right]=h(0) D F(0) \frac{\partial\left(\tilde{F}_{1}, \ldots, \tilde{F}_{n}\right)}{\partial\left(x_{1}, \ldots, x_{n}\right)}(0) .
$$

Now the equality of the residues follows from Lemma 3. 
Proof of Lemma 1. Denote by $\mu$ the local multiplicity of $F$. Let $G$ be defined as above. The regular values of $G$ are dense and we have for those $y=\left(y_{1}, \ldots, y_{n-q}\right)$ with $g_{y}:=\left(g_{1}-y_{1}, \ldots, g_{n-q}-y_{n-q}\right)$

$$
\operatorname{res}_{\mathbb{C}^{n}, 0}\left[\begin{array}{c}
h \cdot D F \\
g_{1} \ldots g_{n-q} f_{1} \ldots f_{q}
\end{array}\right]=\lim _{y \rightarrow 0} \sum_{i=1}^{\mu} \operatorname{res}_{\mathbb{C}^{n}, p_{i}}\left[\begin{array}{c}
h \cdot D F \\
g_{y, 1} \ldots g_{y, n-q} f_{1} \ldots f_{q}
\end{array}\right] .
$$

The value $(y, 0)$ is a regular value of $F$ : The preimages of $y$ under $G$ are $\mu$ simple zeros of $G-y$, which are also the preimages of $(y, 0)$ under $F$. They must be also simple zeros of $F-(y, 0)$ then. By the Claim we get

$$
\operatorname{res}_{\mathbb{C}^{n}, 0}\left[\begin{array}{c}
h \cdot D F \\
g_{1} \ldots g_{n-q} f_{1} \ldots f_{q}
\end{array}\right]=\lim _{y \rightarrow 0} \frac{1}{(2 \pi i)^{n-q}} \int_{\Sigma_{y, \rho}} \frac{h d z_{1} \wedge \cdots \wedge d z_{n-q}}{g_{y, 1} \ldots g_{y, n-q}}
$$

where

$$
\Sigma_{y, \rho}:=\left\{f_{1}=\cdots=f_{q}=0,\left|g_{y, i}\right|=\rho_{i}, i=1, \ldots, n-q\right\}
$$

and $\rho$ is chosen small enough. $\Sigma_{y, \rho}$ decomposes into $\mu$ components then. Now it sufficies to show that $\Sigma_{y, \rho}$ is homologous to $\Sigma$ in

$$
V \backslash\left(\bigcup_{i=1}^{n-q}\left\{g_{i}-y_{i}=0\right\} \cup\{0\}\right) .
$$

This can be done if $y$ is chosen small enough in the same lines as in 11, p. 113].

\section{Residues of holomorphic 1-forms}

To prove our results on holomorphic 1-forms on an isolated surface singularity we will look at the behaviour of the 1-forms on the Milnor fibre. Therefore we first study the smooth case. Then we construct a class $\sigma \in \mathcal{A}$ and show that it plays a similar role the Jacobian plays in the classical case in the algebra $Q_{f}$. The Jacobian generates the 1-dimensional socle of $Q_{f}$ and thus every linear form $l: Q_{f} \rightarrow \mathbb{C}$ which maps the Jacobian not to 0 induces a non-degenerate pairing on $Q_{f}$ (The induced pairing is always the pairing defined by $B(h, g):=l(h \cdot g)$ in this paper). We will define a linear form res: $\mathcal{A} \rightarrow \mathbb{C}$ and use this form to prove a result on the dimension of the socle of $\mathcal{A}$.

\subsection{The smooth case}

Let $(V, 0) \subset\left(\mathbb{C}^{n}, 0\right)$ be an icis as before and $\omega=\sum_{i=1}^{n} \omega_{i} d z_{i}$ the germ of a holomorphic 1-form on $\left(\mathbb{C}^{n}, 0\right)$ with an isolated zero on $(V, 0)$ (does not vanish on the tangent spaces $T_{p} V$ for $p \neq 0$ in a neighbourhood of the origin). For $1 \leq j_{1}, \ldots, j_{q+1} \leq n$ we set

$$
m_{j_{1}, \ldots, j_{q+1}}:=\left|\begin{array}{ccc}
\frac{\partial f_{1}}{\partial z_{j_{1}}} & \ldots & \frac{\partial f_{1}}{\partial z_{j_{q+1}}} \\
\vdots & \ddots & \vdots \\
\frac{\partial f_{q}}{\partial z_{j_{1}}} & \ldots & \frac{\partial f_{q}}{\partial z_{j_{q+1}}} \\
\omega_{j_{1}} & \ldots & \omega_{j_{q+1}}
\end{array}\right| .
$$


Furthermore for $1 \leq i \leq n-q$ we set $\tilde{m}_{i}:=m_{i, n-q+1, \ldots, n}$. Let $J$ be the ideal as in the introduction and $I$ the ideal in $\mathcal{O}_{\mathbb{C}^{n}, 0}$ generated by $f_{1}, \ldots, f_{q}$ and the minors $\tilde{m}_{1}, \ldots, \tilde{m}_{n-q}$. For simplicity we may assume that all minors vanish at the origin (otherwise the index is 0 ).

Lemma 4. For any $i_{1}, \ldots, i_{q}, j_{1}, \ldots, j_{q+1} \in\{1, \ldots, n\}$ we have

$$
\frac{\partial\left(f_{1}, \ldots, f_{q}\right)}{\partial\left(z_{i_{1}}, \ldots, z_{i_{q}}\right)} m_{j_{1}, \ldots, j_{q+1}}=\sum_{l=1}^{q+1}(-1)^{l+1} \frac{\partial\left(f_{1}, \ldots, f_{q}\right)}{\partial\left(z_{j_{1}}, \ldots, \hat{\left.z_{j_{l}}, \ldots, z_{j_{q+1}}\right)}\right.} m_{j_{l}, i_{1}, \ldots, i_{q}} .
$$

Proof. Expansion of $m_{j_{1}, \ldots, j_{q+1}}$ and $m_{j_{l}, i_{1}, \ldots, i_{q}}$ by the last row gives us

$$
\begin{aligned}
\frac{\partial\left(f_{1}, \ldots, f_{q}\right)}{\partial\left(z_{i_{1}}, \ldots, z_{i_{q}}\right)} m_{j_{1}, \ldots, j_{q+1}} & =\sum_{l=1}^{q+1}(-1)^{l+1} \frac{\partial\left(f_{1}, \ldots, f_{q}\right)}{\partial\left(z_{j_{1}}, \ldots, \hat{j_{l}}, \ldots, z_{j_{q+1}}\right)}\left(m_{j_{l}, i_{1}, \ldots, i_{q}}\right. \\
& \left.-\sum_{k=1}^{q}(-1)^{q+k} \omega_{i_{k}} \frac{\partial\left(f_{1}, \ldots, f_{q}\right)}{\partial\left(z_{j_{l}}, z_{i_{1}}, \ldots,{\hat{z_{k}}}_{1}, \ldots, z_{i_{q}}\right)}\right) .
\end{aligned}
$$

Now it sufficies to show for any fixed $k$

$$
\sum_{l=1}^{q+1}(-1)^{l} \frac{\partial\left(f_{1}, \ldots, f_{q}\right)}{\partial\left(z_{j_{1}}, \ldots, \hat{z_{l}}, \ldots, z_{j_{q+1}}\right)} \frac{\partial\left(f_{1}, \ldots, f_{q}\right)}{\partial\left(z_{j_{l}}, z_{i_{1}}, \ldots, \hat{z_{i_{k}}}, \ldots, z_{i_{q}}\right)}=0,
$$

which is obvious by expanding the second determinant by the first column and summing over $l$.

Proposition 1. Let $D F(0) \neq 0$. Then $I=J$.

Proof. For any $j_{1}, \ldots, j_{q+1}$ we get from Lemma $D F m_{j_{1}, \ldots, j_{q+1}} \in I$ and therefore $m_{j_{1}, \ldots, j_{q+1}} \in I$, since $D F$ is a unit.

Now from standard theory we get

Corollary 2. Let $D F(0) \neq 0$. Then

(i) $f_{1}, \ldots, f_{q}, \tilde{m}_{1}, \ldots, \tilde{m}_{n-q}$ defines a regular sequence and

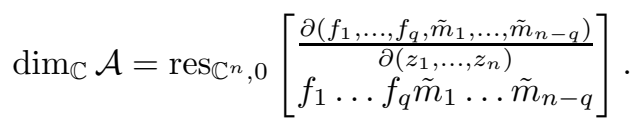

(ii) $\operatorname{res}_{\mathbb{C}^{n}, 0}\left[\begin{array}{c}\cdot \\ f_{1} \ldots f_{q} \tilde{m}_{1} \ldots \tilde{m}_{n-q}\end{array}\right]: \mathcal{A} \rightarrow \mathbb{C}$ defines a linear form.

(iii) The induced pairing on $\mathcal{A}$ is non-degenerate.

\subsection{The index of a holomorphic 1-form}

From now on we restrict to the case $q=n-2$ and we introduce some more notations. Set $m_{i}:=m_{1 \ldots \hat{i} \ldots n}$ and for $l \neq k$

$$
f_{l, k}:=\frac{\partial\left(f_{1}, \ldots, f_{n-2}\right)}{\partial\left(z_{1}, \ldots, \hat{z_{l}}, \ldots, \hat{z_{k}}, \ldots, z_{n}\right)} .
$$


Define $M$ to be the matrix

$$
M:=\frac{\partial\left((-1) m_{1},(-1)^{2} m_{2}, \ldots,(-1)^{n} m_{n}\right)}{\partial\left(z_{1}, \ldots, z_{n}\right)} .
$$

We define $\sigma:=\sigma_{2}(M)$ as a coefficient of the characteristical polynomial of $M$ :

$$
\sum_{i=0}^{n}(-1)^{i} \sigma_{n-i}(M) t^{i}:=\operatorname{det}(M-t I) .
$$

We now want to prove a refinement of Corollary 2 that will be used to prove our main result.

Lemma 5. Let $(V, 0)$ be smooth and assume $m_{1}, m_{2}$ to be a regular $\mathcal{O}_{V, 0^{-}}$ sequence. Then

$$
\operatorname{dim}_{\mathbb{C}} \mathcal{A}=\operatorname{res}_{\mathbb{C}^{n}, 0}\left[\begin{array}{c}
D F \cdot \sigma \\
f_{1} \ldots f_{n-2} m_{1} m_{2}
\end{array}\right]
$$

Note that we have not assumed $D F(0) \neq 0$. In order to prove this we need a computation.

Lemma 6. For $j<k$ we have

$$
\frac{\partial\left(f_{1}, \ldots, f_{n-2}, m_{j}, m_{k}\right)}{\partial\left(z_{1}, \ldots, z_{n}\right)}=-f_{j, k} \cdot \sigma \bmod J .
$$

Proof. The expansion formula gives us

$$
\frac{\partial\left(f_{1}, \ldots, f_{n-2}, m_{j}, m_{k}\right)}{\partial\left(z_{1}, \ldots, z_{n}\right)}=\sum_{1 \leq m<l \leq n}(-1)^{m+l+1} f_{m, l} \frac{\partial\left(m_{j}, m_{k}\right)}{\partial\left(z_{m}, z_{l}\right)} .
$$

Now it sufficies to prove $\bmod J$

$$
f_{m, l} \frac{\partial\left(m_{j}, m_{k}\right)}{\partial\left(z_{m}, z_{l}\right)}=f_{j, k} \frac{\partial\left(m_{m}, m_{l}\right)}{\partial\left(z_{m}, z_{l}\right)} .
$$

The first case is $m, l<j$. By Lemma 0 we get $\bmod J$

$$
\begin{aligned}
f_{m, l} \frac{\partial\left(m_{j}, m_{k}\right)}{\partial\left(z_{m}, z_{l}\right)} & =f_{m, j} \frac{\partial\left(m_{l}, m_{k}\right)}{\partial\left(z_{m}, z_{l}\right)}-f_{l, j} \frac{\partial\left(m_{m}, m_{k}\right)}{\partial\left(z_{m}, z_{l}\right)} \\
& =f_{m, k} \frac{\partial\left(m_{l}, m_{j}\right)}{\partial\left(z_{m}, z_{l}\right)}-f_{j, k} \frac{\partial\left(m_{l}, m_{m}\right)}{\partial\left(z_{m}, z_{l}\right)} \\
& -f_{l, k} \frac{\partial\left(m_{m}, m_{j}\right)}{\partial\left(z_{m}, z_{l}\right)}+f_{j, k} \frac{\partial\left(m_{m}, m_{l}\right)}{\partial\left(z_{m}, z_{l}\right)}
\end{aligned}
$$

On the other hand we get again by Lemma $1 \bmod J$

$$
f_{m, l} \frac{\partial\left(m_{j}, m_{k}\right)}{\partial\left(z_{m}, z_{l}\right)}=f_{m, k} \frac{\partial\left(m_{j}, m_{l}\right)}{\partial\left(z_{m}, z_{l}\right)}-f_{l, k} \frac{\partial\left(m_{j}, m_{m}\right)}{\partial\left(z_{m}, z_{l}\right)} .
$$

Summing the two equations we get the Lemma in this case. All other cases can be proved similarly. 
Proof of Lemma 5. Let $k<l$ with $f_{k, l}(0) \neq 0$. By a trivial generalization of Corollary 2 we get

$$
\operatorname{dim}_{\mathbb{C}} \mathcal{A}=\operatorname{res}_{\mathbb{C}^{n}, 0}\left[\frac{\partial\left(f_{1}, \ldots, f_{n-2}, m_{k}, m_{l}\right)}{\partial\left(z_{1}, \ldots, z_{n}\right)}\right] .
$$

The first and most complicated case is $3 \leq k$. Here we get by Lemma 1

$$
\begin{aligned}
& f_{k, l} m_{1}=-f_{1, k} m_{l}+f_{1, l} m_{k} \\
& f_{k, l} m_{2}=-f_{2, k} m_{l}+f_{2, l} m_{k} .
\end{aligned}
$$

Furthermore it is not hard to compute

$$
f_{2, k} f_{1, l}-f_{1, k} f_{2, l}=-f_{k, l} D F
$$

and therefore we get by the transformation formula for residues and Lemma 6

$$
\begin{aligned}
\operatorname{dim}_{\mathbb{C}} \mathcal{A} & =\operatorname{res}_{\mathbb{C}^{n}, 0}\left[\begin{array}{c}
\frac{-D F}{f_{l, k}} \frac{\partial\left(f_{1}, \ldots, f_{n-2}, m_{k}, m_{l}\right)}{\partial\left(z_{1}, \ldots, z_{n}\right)} \\
f_{1} \ldots f_{n-2} m_{1} m_{2}
\end{array}\right] \\
& =\operatorname{res}_{\mathbb{C}^{n}, 0}\left[\begin{array}{c}
D F \cdot \sigma \\
f_{1} \ldots f_{n-2} m_{1} m_{2}
\end{array}\right]
\end{aligned}
$$

using that the product of $D F$ and any minor is contained in $I$. All other cases can be proved similarly.

Now we generalize to the singular case.

Lemma 7. There exists a linear change of coordinates so that $m_{1}, m_{2}$ is a regular $\mathcal{O}_{V, 0}$-sequence.

Proof. Let $\phi:\left(\mathbb{C}^{n}, 0\right) \rightarrow\left(\mathbb{C}^{n}, 0\right)$ be biholomorphic with $\phi(y)=z$ and set $\psi:=$ $\phi^{-1}$. Denote by $m_{i}^{y}$ the minors computed in $y$-coordinates. By standard computations we get for $i=1, \ldots, n$

$$
\begin{aligned}
m_{i}^{y} & =\sum_{j=1}^{n} \frac{\partial\left(\phi_{1}, \ldots, \hat{\phi}_{j}, \ldots, \phi_{n}\right)}{\partial\left(y_{1}, \ldots, \hat{y}_{i}, \ldots, y_{n}\right)} m_{j} \circ \phi \\
& =\sum_{j=1}^{n}(-1)^{i+j} \operatorname{det} D \phi \frac{\partial \psi_{i}}{\partial z_{j}} \circ \phi \cdot m_{j} \circ \phi .
\end{aligned}
$$

Since $\mathcal{O}_{V, 0}$ is a complete intersection there are complex numbers $c_{11}, \ldots, c_{1 n}$, $c_{21}, \ldots, c_{2 n}$ so that $g_{1}, g_{2}$ is a regular $\mathcal{O}_{V, 0^{-}}$-sequence where $g_{1}:=\sum_{i=1}^{n} c_{1 i} m_{i}$ and $g_{2}:=\sum_{i=1}^{n} c_{2 i} m_{i}$ and therefore $\phi^{*}\left(f_{1}\right), \ldots, \phi^{*}\left(f_{n-2}\right), \phi^{*}\left(g_{1}\right), \phi^{*}\left(g_{2}\right)$ is a regular $\mathcal{O}_{\mathbb{C}^{n}, 0^{-}}$-sequence. Since the vectors $\left(c_{11}, \ldots, c_{1 n}\right)$ and $\left(c_{21}, \ldots, c_{2 n}\right)$ are linearly independent we can extend them to a squared matrix of complex numbers $C$ with $\operatorname{det} C=1$. Define $C^{\prime}$ to be the matrix with entries $c_{i j}^{\prime}:=(-1)^{i+j} c_{i j}$. Then $\operatorname{det} C^{\prime}=1$ and therefore $C^{\prime}$ defines a biholomorphic map. By equation 3 it sufficies now to set $\phi:=\left(C^{\prime}\right)^{-1}$ since we have $\phi^{*}\left(g_{1}\right)=m_{1}^{y}$ resp. $\phi^{*}\left(g_{2}\right)=$ $m_{2}^{y}$. 
We call a system of coordinates as in the Lemma a good system of coordinates. Further we define for good coordinates

$$
\operatorname{res}_{V, 0}\left[\begin{array}{c}
h \\
m_{1} m_{2}
\end{array}\right]:=\operatorname{res}_{V, 0}\left[\begin{array}{c}
h d z_{1} \wedge d z_{2} \\
m_{1} m_{2}
\end{array}\right] .
$$

Now we can easily prove our main result.

Proof of Theorem 3. Since the product of DF and any minor is contained in $I$ the residue defines obviously a linear form on $\mathcal{A}$ by Lemma 1. On the other hand we have

$$
\begin{aligned}
\operatorname{res}_{V, 0}\left[\begin{array}{c}
\sigma \\
m_{1} m_{2}
\end{array}\right] & =\operatorname{res}_{\mathbb{C}^{n}, 0}\left[\begin{array}{c}
D F \sigma \\
f_{1} \ldots f_{n-2} m_{1} m_{2}
\end{array}\right] \\
& =\lim _{\epsilon \rightarrow 0} \sum_{i} \operatorname{res}_{\mathbb{C}^{n}, p_{i}}\left[\begin{array}{c}
D F \sigma \\
f_{\epsilon, 1} \ldots f_{\epsilon, n-2} m_{1} m_{2}
\end{array}\right]
\end{aligned}
$$

where $f_{\epsilon}:=\left(f_{1}-\epsilon_{1}, \ldots, f_{n-2}-\epsilon_{n-2}\right)$ and we sum over the zeros of $\left(m_{1}, m_{2}\right)$ on the Milnor fibre $V_{\epsilon}$. We may first ask if $\left(m_{1}, m_{2}\right)$ can have a zero $p_{i}$ on $V_{\epsilon}$ when $\operatorname{ind}_{V_{\epsilon, p_{i}}} \omega=0$. In this case let $f_{l, k}\left(p_{i}\right) \neq 0$ and we find

$$
\frac{\partial\left(f_{\epsilon, 1}, \ldots, f_{\epsilon, n-2}, m_{l}, m_{k}\right)}{\partial\left(z_{1}, \ldots, z_{n}\right)} \in \mathcal{O}_{\mathbb{C}^{n}, p_{i}}\left(f_{\epsilon, 1}, \ldots, f_{\epsilon, n-2}, m_{l}, m_{k}\right)
$$

since one of the minors $m_{l}, m_{k}$ does not vanish in $p_{i}$. Cramers rule and the same matrix transformation as in the proof of Lemma 5 show that $D F \sigma \in$ $\mathcal{O}_{\mathbb{C}^{n}, p_{i}}\left(f_{\epsilon, 1}, \ldots, f_{\epsilon, n-2}, m_{1}, m_{2}\right)$. This means that the residue vanishes in such a point $p_{i}$. Therefore the above sum of residues is the sum of the indices of $\omega$ on the Milnor fibre by Lemma 5 which equals $\operatorname{dim}_{\mathbb{C}} \mathcal{A}$ by the theorem of Ebeling and Gusein-Zade.

We cannot expect that the induced pairing is non-degenerate because $\mathcal{A}$ in general has no 1-dimensional socle and therefore there cannot exist a nondegenerate pairing induced by a linear form. We go into more detail in the next subsection.

\subsection{Properties of the residue form}

We want to show that the rank of the induced pairing $\beta$ on $\mathcal{A}$ doesn't depend on the choice of a good system of coordinates. Let $\phi:\left(\mathbb{C}^{n}, 0\right) \rightarrow\left(\mathbb{C}^{n}, 0\right)$ be biholomorphic, $\psi:=\phi^{-1}$ and $\phi(y)=z$. We denote by $m_{i}^{y}$ the minors computed in $y$-coordinates and similarly

$$
D F^{y}:=\frac{\partial\left(f_{1} \circ \phi, \ldots, f_{n-2} \circ \phi\right)}{\partial\left(y_{3}, \ldots, y_{n}\right)} .
$$

Lemma 8. Let $\left(m_{1}, m_{2}\right)$ and $\left(m_{1}^{y}(\psi), m_{2}^{y}(\psi)\right)$ be regular $\mathcal{O}_{V, 0}$-sequences. Then for any $h \in \mathcal{O}_{\mathbb{C}^{n}, 0}$

$$
\operatorname{res}_{\mathbb{C}^{n}, 0}\left[\begin{array}{c}
h D F \\
f_{1} \ldots f_{n-2} m_{1} m_{2}
\end{array}\right]=\operatorname{res}_{\mathbb{C}^{n}, 0}\left[\begin{array}{c}
\operatorname{det}(D \phi)(\psi) h D F^{y}(\psi) \\
f_{1} \ldots f_{n-2} m_{1}^{y}(\psi) m_{2}^{y}(\psi)
\end{array}\right] .
$$


Proof. The first step is to show the equality if $(V, 0)$ is smooth. Let $k<l$ with $f_{k, l}(0) \neq 0$. In the same way as in the proof of Lemma 5 we get

$$
\operatorname{res}_{\mathbb{C}^{n}, 0}\left[\begin{array}{c}
h D F \\
f_{1} \ldots f_{n-2} m_{1} m_{2}
\end{array}\right]=\operatorname{res}_{\mathbb{C}^{n}, 0}\left[\begin{array}{c}
h f_{k, l} \\
f_{1} \ldots f_{n-2} m_{k} m_{l}
\end{array}\right] .
$$

Lemma 4 shows now

$$
f_{k, l} m_{j}=\left\{\begin{aligned}
-f_{j, k} m_{l}+f_{j, l} m_{k} & \text { for } j<k \\
f_{j, k} m_{l}+f_{j, l} m_{k} & \text { for } k<j<l \\
f_{j, k} m_{l}-f_{j, l} m_{k} & \text { for } l<j
\end{aligned}\right.
$$

Using this and equation 3 we get

$$
\begin{aligned}
f_{k, l} m_{1}^{y}(\psi) & =\operatorname{det}(D \phi)(\psi) m_{k}\left(\sum_{j=1}^{l-1}(-1)^{j+1} \frac{\partial \psi_{1}}{\partial z_{j}} f_{j, l}+\sum_{j=l+1}^{n}(-1)^{j} \frac{\partial \psi_{1}}{\partial z_{j}} f_{j, l}\right) \\
& +\operatorname{det}(D \phi)(\psi) m_{l}\left(\sum_{j=1}^{k-1}(-1)^{j} \frac{\partial \psi_{1}}{\partial z_{j}} f_{j, k}+\sum_{j=k+1}^{n}(-1)^{j+1} \frac{\partial \psi_{1}}{\partial z_{j}} f_{j, k}\right) \\
f_{k, l} m_{2}^{y}(\psi) & =\operatorname{det}(D \phi)(\psi) m_{k}\left(\sum_{j=1}^{l-1}(-1)^{j} \frac{\partial \psi_{2}}{\partial z_{j}} f_{j, l}+\sum_{j=l+1}^{n}(-1)^{j+1} \frac{\partial \psi_{2}}{\partial z_{j}} f_{j, l}\right) \\
& +\operatorname{det}(D \phi)(\psi) m_{l}\left(\sum_{j=1}^{k-1}(-1)^{j+1} \frac{\partial \psi_{2}}{\partial z_{j}} f_{j, k}+\sum_{j=k+1}^{n}(-1)^{j} \frac{\partial \psi_{2}}{\partial z_{j}} f_{j, k}\right) .
\end{aligned}
$$

Therefore we have a matrix $A$ with

$$
\left(\begin{array}{c}
f_{k, l} m_{1}^{y}(\psi) \\
f_{k, l} m_{2}^{y}(\psi)
\end{array}\right)=A\left(\begin{array}{c}
m_{k} \\
m_{l}
\end{array}\right)
$$

Using the formula

$$
f_{i, l} f_{j, k}= \pm f_{k, l} f_{i, j} \pm f_{j, l} f_{i, k}
$$

where $i, l, k, k$ are pairwise disjoint and the signs depend on the position of the indices, it is not hard to compute

$$
\begin{aligned}
\operatorname{det} A & =\operatorname{det}(D \phi)^{2}(\psi) f_{k, l} \sum_{1 \leq i<j \leq n}(-1)^{i+j+1} \frac{\partial\left(\psi_{1}, \psi_{2}\right)}{\partial\left(z_{i}, z_{j}\right)} f_{i, j} \\
& =\operatorname{det}(D \phi)(\psi) f_{k, l} D F^{y}(\psi) .
\end{aligned}
$$

Application of the transformation formula for residues finishes the proof in the smooth case. To generalize to the singular case we have to show

$$
\sum_{i} \operatorname{res}_{\mathbb{C}^{n}, p_{i}}\left[\begin{array}{c}
h D F \\
f_{\epsilon, 1} \ldots f_{\epsilon, n-2} m_{1} m_{2}
\end{array}\right]=\sum_{j} \operatorname{res}^{n}, q_{j}\left[\begin{array}{c}
\operatorname{det}(D \phi)(\psi) h D F^{y}(\psi) \\
f_{\epsilon, 1} \ldots f_{\epsilon, n-2} m_{1}^{y}(\psi) m_{2}^{y}(\psi)
\end{array}\right],
$$

where the first sum is taken over the zeros of $g_{1}:=\left(m_{1}, m_{2}\right)$ on the Milnor fibre $V_{\epsilon}$ and the second over the zeros of $g_{2}:=\left(m_{1}^{y}(\psi), m_{2}^{y}(\psi)\right)$ on $V_{\epsilon}$. The residues equal each other at those points which are common zeros of $g_{1}$ and $g_{2}$. Let $p_{i}$ be a zero of $g_{1}$ with $g_{2}\left(p_{i}\right) \neq 0$. Then $\operatorname{ind}_{V_{\epsilon}, p_{i}} \omega=0$. If $f_{l, k}\left(p_{i}\right) \neq 0$ 
we have $h f_{k, l} \in \mathcal{O}_{\mathbb{C}^{n}, p_{i}}\left(f_{\epsilon, 1}, \ldots, f_{\epsilon, n-2}, m_{1}, m_{2}\right)$. The same transformation as at the beginning of the proof and application of Cramers rule show $h D F \in$ $\mathcal{O}_{\mathbb{C}^{n}, p_{i}}\left(f_{\epsilon, 1}, \ldots, f_{\epsilon, n-2}, m_{1}, m_{2}\right)$. Therefore the residue vanishes at such a point. Similarly one arguments for a zero $q_{j}$ of $g_{2}$ with $g_{1}\left(q_{j}\right) \neq 0$. Since the above sums of residues are equal the limit procedure gives us the equality of the residues at the origin.

We assume that the chosen coordinates as well as the $y$-coordinates are good. We define

$$
\mathcal{B}:=\frac{\mathcal{O}_{V, 0}}{\left(m_{1}, m_{2}\right)}, \quad \mathcal{C}:=\frac{\mathcal{B}}{\operatorname{ann}_{\mathcal{B}}(D F)} .
$$

Since we have $\mathcal{B}\left(m_{3}, \ldots, m_{n}\right) \subset \operatorname{ann}_{\mathcal{B}}(D F)$ we find that $\operatorname{dim}_{\mathbb{C}} \mathcal{C} \leq \operatorname{dim}_{\mathbb{C}} \mathcal{A}$ and the residue induces a linear form on $\mathcal{C}$, the induced pairing on $\mathcal{C}$ is nondegenerate, since

$$
\operatorname{res}_{V, 0}\left[\begin{array}{c}
h g \\
m_{1} m_{2}
\end{array}\right]=0 \text { for all } g \in \mathcal{O}_{\mathbb{C}^{n}, 0}
$$

implies

$$
\operatorname{res}_{\mathbb{C}^{n}, 0}\left[\begin{array}{c}
h D F g \\
f_{1} \ldots f_{n-2} m_{1} m_{2}
\end{array}\right]=0 \text { for all } g \in \mathcal{O}_{\mathbb{C}^{n}, 0}
$$

By duality we get $h D F \in I$ and therefore $h \in \operatorname{ann}_{\mathcal{B}}(D F)$. This means that $\mathcal{C}$ has an 1-dimensional socle socC. It is generated by the class of $\sigma$ : Let $g(0)=0$. Then we get

$$
\begin{aligned}
\operatorname{res}_{V, 0}\left[\begin{array}{c}
g \sigma \\
m_{1} m_{2}
\end{array}\right] & =\lim _{\epsilon \rightarrow 0} \sum_{i} \operatorname{res}_{\mathbb{C}^{n}, p_{i}}\left[\begin{array}{c}
g D F \sigma \\
f_{\epsilon, 1} \ldots f_{\epsilon, n-2} m_{1} m_{2}
\end{array}\right] \\
& =\lim _{\epsilon \rightarrow 0} \sum_{i} g\left(p_{i}\right) \operatorname{ind}_{V_{\epsilon}, p_{i}} \omega \\
& =0
\end{aligned}
$$

and therefore $\sigma \in \operatorname{soc} \mathcal{C}$. Furthermore we find $\operatorname{rank} \beta=\operatorname{dim}_{\mathbb{C}} \mathcal{C}$ and we can conclude

$$
\operatorname{dim}_{\mathbb{C}} \operatorname{soc} \mathcal{A} \leq \operatorname{dim}_{\mathbb{C}} \mathcal{A}-\operatorname{dim}_{\mathbb{C}} \mathcal{C}+1 .
$$

We want to show that $\operatorname{dim}_{\mathbb{C}} \mathcal{C}$ does not depend on the choice of a good system of coordinates. Since we have an exact sequence

$$
0 \rightarrow \operatorname{ann}_{\mathcal{B}}(D F) \rightarrow \mathcal{B} \stackrel{\cdot D F}{\rightarrow} \mathcal{B} \rightarrow \frac{\mathcal{B}}{\mathcal{B}(D F)} \rightarrow 0
$$

we get $\operatorname{dim}_{\mathbb{C}} \mathcal{C}=\operatorname{dim}_{\mathbb{C}} \mathcal{B}(D F)$. Let

$$
\mathcal{B}^{\prime}:=\frac{\mathcal{O}_{\mathbb{C}^{n}, 0}}{\left(f_{1}(\phi), \ldots, f_{n-2}(\phi), m_{1}^{y}, m_{2}^{y}\right)} .
$$

We have to show $\operatorname{dim}_{\mathbb{C}} \mathcal{B}(D F)=\operatorname{dim}_{\mathbb{C}} \mathcal{B}^{\prime}\left(D F^{y}\right)$. Since we have

$$
\operatorname{dim}_{\mathbb{C}} \mathcal{B}^{\prime}\left(D F^{y}\right)=\operatorname{dim}_{\mathbb{C}} \mathcal{B}^{\prime \prime}\left(D F^{y}(\psi)\right)
$$


with

$$
\mathcal{B}^{\prime \prime}:=\frac{\mathcal{O}_{\mathbb{C}^{n}, 0}}{\left(f_{1}, \ldots, f_{n-2}, m_{1}^{y}(\psi), m_{2}^{y}(\psi)\right)}
$$

it remains to construct an isomorphism of vector spaces

$$
\varphi: \mathcal{B}(D F) \rightarrow \mathcal{B}^{\prime \prime}\left(D F^{y} \circ \psi\right) .
$$

We set $\varphi(g D F):=g D F^{y}(\psi)$. Now Lemma 8 and duality show that

$$
g \in \operatorname{ann}_{\mathcal{B}}(D F) \Longleftrightarrow g \in \operatorname{ann}_{\mathcal{B}^{\prime \prime}}\left(D F^{y}(\psi)\right)
$$

which means that $\varphi$ is well defined and injective and of course surjective. We want to summarize:

Proposition 2. (i) The residue induces a non-degenerate pairing on $\mathcal{C}$.

(ii) The 1-dimensional socle of $\mathcal{C}$ is generated by $\sigma$.

(iii) The dimension of $\mathcal{C}$ does not depend on the choice of a good system of coordinates.

(iv) $\operatorname{rank} \beta=\operatorname{dim}_{\mathbb{C}} \mathcal{C}$, in particular if $V$ is smooth then $\beta$ is non-degenerate and $\sigma$ generates the 1-dimensional socle of $\mathcal{A}$.

(v) $\operatorname{dim}_{\mathbb{C}} \operatorname{soc} \mathcal{A} \leq \operatorname{dim}_{\mathbb{C}} \mathcal{A}-\operatorname{dim}_{\mathbb{C}} \mathcal{C}+1$.

\section{Remarks}

\subsection{The case of curve singularities}

We have only one minor $m$ here and therefore the algebra $\mathcal{A}$ is a complete intersection. Residue theory can be applied directly so that we obtain a nondegenerate pairing on $\mathcal{A}$. But the dimension of $\mathcal{A}$ can also be expressed by a relative integral

$$
\operatorname{dim}_{\mathbb{C}} \mathcal{A}=\frac{1}{2 \pi i} \int_{\Sigma} \frac{d m}{m}
$$

by Corollary 1 .

\subsection{The general case}

We may ask how to generalize our results when we consider a general icis. The problem is that in general there is no choice of coordinates so that $\left(\tilde{m}_{1}, \ldots, \tilde{m}_{n-q}\right)$ is a regular $\mathcal{O}_{V, 0}$-sequence and therefore the residues don't exist. There is a simple reason that was pointed out to the author by S.M. Gusein-Zade. Let $n>2 q+1$. Assume that $f_{1}, \ldots, f_{q}, \tilde{m}_{1}, \ldots, \tilde{m}_{n-q}$ define an isolated zero. Consider the $(q+1)$ maximal minors of the matrix

$$
\left(\begin{array}{ccc}
\frac{\partial f_{1}}{\partial z_{n-q+1}} & \cdots & \frac{\partial f_{1}}{\partial z_{n}} \\
\vdots & \ddots & \vdots \\
\frac{\partial f_{q}}{\partial z_{n-q+1}} & \cdots & \frac{\partial f_{q}}{\partial z_{n}} \\
\omega_{n-q+1} & \cdots & \omega_{n}
\end{array}\right) .
$$

Then the vanishing of these minors at the origin would imply that $2 q+1$ equations define an isolated zero which is not possible. If one of these minors is a unit in $\mathcal{O}_{\mathbb{C}^{n}, 0}$, then $\mathcal{A}$ is a complete intersection. 


\subsection{Linear forms on spaces of relative holomorphic forms}

It also seems to be natural to define linear forms on the vector space $\Omega_{V, 0}^{n-q} / \omega \wedge$ $\Omega_{V, 0}^{n-q-1}$ since it's dimension is also ind ${ }_{V, 0} \omega$ : We have an exact sequence

$$
0 \longrightarrow T \Omega_{V, 0}^{n-q} \longrightarrow \frac{\Omega_{V, 0}^{n-q}}{\omega \wedge \Omega_{V, 0}^{n-q-1}} \stackrel{\lambda}{\longrightarrow} \frac{J^{\prime}}{J} \longrightarrow 0
$$

and $\operatorname{dim}_{\mathbb{C}} T \Omega_{V, 0}^{n-q}=\operatorname{dim}_{\mathbb{C}} \mathcal{O}_{\mathbb{C}^{n}, 0} / J^{\prime}$ where $T \Omega_{V, 0}^{n-q}$ is the torsion submodule and $J^{\prime}$ the ideal in $\mathcal{O}_{\mathbb{C}^{n}, 0}$ generated by the components of $f$ and the maximal minors of it's Jacobi matrix. Details of this argumentation can be found in [6]. If we have a regular $\mathcal{O}_{V, 0}$-sequence $\left(g_{1}, \ldots, g_{n-q}\right)$ and the residue $\operatorname{res}_{V, 0}\left[\begin{array}{c}\cdot \\ g_{1} \ldots g_{n-q}\end{array}\right]$ vanishes on $\omega \wedge \Omega_{V, 0}^{n-q-1}$ the absolute residue $\operatorname{res}_{\mathbb{C}^{n}, 0}\left[\begin{array}{c}\cdot \\ g_{1} \ldots g_{n-q} f_{1} \ldots f_{q}\end{array}\right]$ vanishes on $J$ since $m_{j_{1}, \ldots, j_{q+1}} \in \lambda\left(\omega \wedge \Omega_{V, 0}^{n-q-1}\right)$ for each minor.

\section{References}

[1] V.I. Arnold, S.M. Gusein-Zade, A.N. Varchenko: Singularities of Differentiable Maps. I. Birkhäuser-Verlag, Basel, 1985

[2] Ch. Bonatti and X. Gomez-Mont: The index of a holomorphic vector field on a singular variety I. Asterisque 222, (1994) 9-35

[3] W. Ebeling and S.M. Gusein-Zade: On the Index of a Holomorphic 1-Form on an Isolated Complete Intersection Singularity. Doklady Mathematics 64, No. 2, (2001) 221-224

[4] X. Gomez-Mont: An algebraic formula for the index of a vector field on a hypersurface with an isolated singularity. J. of Alg. Geom. 7, (1998) 731-752

[5] X. Gomez-Mont, J. Seade, and A. Verjowsky: The index of a holomorphic flow with an isolated singularity. Math. Ann. 291, (1991) 737-751

[6] G.-M. Greuel: Der Gauß-Manin Zusammenhang isolierter Singularitäten von vollständigen Durchschnitten. Math. Ann. 214, (1975) 235-266

[7] Ph. Griffiths and J. Harris: Principles of Algebraic Geometry. J. Wiley and Sons, New York, 1978

[8] D. Lehmann and T. Suwa: Residues of holomorphic vector fields relative to singular invariant subvarieties. J. Differential Geometry 42, (1995) 165-192

[9] D. Lehmann and T. Suwa: Residues of holomorphic vector fields on singular varieties. Ecuaciones Diferenciales Singularidades, ed. J. Mozo Fernandes, Universidad de Valladolid (1997) 159-182

[10] D. Lehmann, M. Soares, and T. Suwa: On the index of a holomorphic vector field tangent to a singular variety. Bol. Soc. Bras. Mat. 26, (1995) 183-199 
[11] John W. Milnor: Topology from the Differentiable Viewpoint. University Press of Virginia, 1965

[12] J.A. Seade and T. Suwa: A residue formula for the index of a holomorphic flow. Math. Ann. 304, (1996) 621-634 